\title{
A new species of Paracapillaroides (Nematoda: Capillariidae) parasitizing Nemadactylus bergi (Perciformes: Latridae) from the Argentine Sea
}

\author{
María Alejandra Rossin and Juan Tomás Timi
}

Laboratorio de Parasitología, Departamento de Biología, Facultad de Ciencias Exactas y Naturales, Universidad Nacional de Mar del Plata - Consejo Nacional de Investigaciones Científicas y Técnicas (CONICET), Funes 3350, (7600) Mar del Plata, Argentina

\begin{abstract}
A new nematode species, Paracapillaroides acanthocotylus sp. n., is described from the marine fish Nemadactylus bergi (Norman) (Latridae, Perciformes) from waters off Mar del Plata, Argentina (38 $08^{\circ} \mathrm{S}, 57^{\circ} 32^{\prime} \mathrm{W}$ ) (prevalence $81.8 \%$, mean intensity $12.4 \pm 10.3$ ). The new species is readily distinguished from P. agonostomi Moravec, Salgado-Maldonado et Caspeta-Mandujano, 1999, the only known species of the genus, by having in both sexes a longer oesophagus in relation to total body length. Males of the new species have a shorter and more complex spicule and a markedly different morphology of the spicular sheath; the rays supporting the caudal bursa are also shorter and rounded, instead of digitiform. Furthermore, females of the new species have elevated vulval lips and longer eggs. The complex structure of both the spicule and spicular sheath is unique among all capillariids parasitizing cold-blooded vertebrates. This is the first record of a species of Paracapillaroides in the southwestern Atlantic Ocean.
\end{abstract}

Key words: parasitic nematodes, Paracapillaroides, new species, marine fish, Argentine Sea

Species of the nematode family Capillariidae Railliet, 1915 have a worldwide distribution, with representatives of 14 genera parasitizing cold-blooded vertebrates (Moravec 2001). Two species of this family have recently been described from fishes inhabiting the Argentine Sea, Capillaria (Procapillaria) navoneae Timi, Rossin et Lanfranchi, 2006, an intestinal parasite of the conger Conger orbignianus Valenciennes and Paracapillaria (Paracapillaria) argentinensis Timi, Rossin, Lanfranchi et Etchegoin, 2007, a parasite of the stomach of the sandperch Pinguipes brasilianus Cuvier (see Timi et al. 2006, 2007).

During a parasitological survey carried out on samples of Nemadactylus bergi (Norman) (syn. Cheilodactylus bergi), landed by fishermen at Mar del Plata port, Argentina, capillariid nematodes referable to Paracapillaroides Moravec, Salgado-Maldonado et Caspeta-Mandujano, 1999 were found in the stomach of these fishes. Paracapillaroides is a monotypic genus that closely resembles Paracapillaria Mendoça, 1963 in its general morphology, but differs conspicuously from it by the morphology of its spicular sheath. While the spicular sheath of Paracapillaria is undivided and non-spiny, Paracapillaroides has a spicular sheath divided into two distinct portions, a proximal part covered with unevenly distributed spines of different size and a non-spiny distal part (Moravec et al. 1999). The structure of the spicular sheath of Paracap- illaroides and the characteristics of its spination appear to be unique among capillariids (Moravec 2001). The only known species of this genus, P. agonostomi Moravec, Salgado-Maldonado et Caspeta-Mandujano, 1999, parasitizes the stomach of Agonostomus monticola Bancroft (Mugilidae, Perciformes) in the Cuitzmala River, Chamela, Mexico.

The newly collected specimens are described herein and a new species is proposed for them.

\section{MATERIALS AND METHODS}

A total of 33 specimens of Nemadactylus bergi caught by commercial trawlers operating at the Mar del Plata port $\left(38^{\circ} 08^{\prime} \mathrm{S}, 57^{\circ} 32^{\prime} \mathrm{W}\right)$, during May to June 2008 , were examined for nematodes immediately after capture. Fish were dissected, and the stomachs were removed and examined under a stereoscopic microscope. A total of 328 capillariid nematodes was collected, fixed in 4\% formaldehyde solution and preserved in $70 \%$ ethanol, then studied and measured by light microscopy. Drawings were made using a drawing tube. All measurements are given in micrometres unless otherwise indicated as the mean followed by range in parentheses. The studied material was deposited in the Colección Helmintológica del Museo de La Plata (C.H.M.L.P.), La Plata, Argentina and in the Helminthological Collection of the Institute of Parasitology, Biology Centre of ASCR in České Budějovice, Czech Republic.

Address for correspondence: M.A. Rossin, Laboratorio de Parasitología, Departamento de Biología, Facultad de Ciencias Exactas y Naturales, Universidad Nacional de Mar del Plata, Funes 3350, (7600) Mar del Plata, Argentina. Phone: +54 0223 475-4060; Fax: +54 0223 475-3150; E-mail: mrossin@mdp.edu.ar 


\section{RESULTS}

Paracapillaroides acanthocotylus sp. n. Figs. 1-10

General: Small-sized nematodes; males smaller than females. Cephalic end narrow and rounded. Cephalic papillae indistinct. Cuticle smooth. Two lateral bacillary bands extending along whole body. Nerve ring in first third of muscular oesophagus. Muscular oesophagus-stichosome junction oblique. Stichosome arranged in single row of 24-39 stichocytes, each bearing a medium-sized, irregular, central nucleus. Stichocytes differing in size from 50 to 175 long, each subdivided in approximately 7-10 annuli; lighter-coloured stichocytes alternating with shorter and darker ones. Two large glandular cells present at oesophago-intestinal junction.

Male (10 specimens): Body 3.16 (2.70-3.60) mm long and 39 (35-45) maximum wide. Bacillary bands 11 (10 12) wide at middle region of body. Nerve ring situated at 115 (96-130) from apex. Muscular oesophagus 172 (163-180) long; stichosome 2.02 (1.68-2.36) mm long, composed of 29 (24-34) stichocytes; entire oesophagus 2.19 (1.85-2.52) $\mathrm{mm}$ long, representing 69 (65-75)\% of body length. Length forebody/hindbody ratio $1: 2.3$. Testis reaches anteriorly to level of oesophago-intestinal junction. Seminal vesicle not differentiated from vas deferens and testis, ejaculatory duct oval, sac-like, 40-57 long. Intestine joins cloaca immediately posterior to ejaculatory duct. Cloaca 294 (268-332) long, representing 9.5 (8.2$11.6) \%$ of body length, anterior cloaca 138 (88-190) long, posterior cloaca 162 (140-185) long. Spicule well sclerotized and sigmoid, with expanded proximal end provided with irregular rim; proximal part wrinkled, followed by expansion separated by constriction from blade-like distal end. Spicule total length 115 (96-130), representing 3.7 (3.1-4.8)\% of body length, $12(10-15)$ wide at expanded proximal end, 5 (5-6) wide in expanded anterior third, 3 (2-3) wide at middle constriction and 5 (4-6) wide at distal end. Spicular canal not developed. Spicular sheath complex, 90-95 long when fully evaginated (observed in 2 specimens), consisting of two different parts: shorter spinose proximal part and longer, non-spiny distal part. Proximal part cup-shaped, broadening distally and forming ventral concavity at its distal end; latter surrounded by cuticularized rim, whereas basal part covered by minute scales at its anterior part which become true spines distally. Dorsally to concavity, basal part continues as cylindrical and sinuous tube provided with ventral field of spines. Distal part of spicular sheath as narrow tube slightly striated. Caudal end rounded, bearing well-developed cuticular membranous bursa supported by 2 lateral rays bent along posterior border of bursa to dorsal side. Dorsal caudal projection absent. One pair of medium sized adanal papillae present at base of caudal projections. Tail 12 (10-15) long.
Female (10 specimens): Body $6.41(5.68-7.00) \mathrm{mm}$ long and 68 (60-83) maximum wide. Bacillary bands 18 (15-20) wide at level of vulva. Nerve ring situated at 91 (75-100) from apex. Muscular oesophagus 224 (205-245) long; stichosome 3.35 (3.00-3.67) mm long, composed of 34 (30-39) stichocytes; entire oesophagus 3.57 (3.24-3.90) $\mathrm{mm}$ long, representing 56 (50-60)\% of body length. Length forebody/hindbody ratio $1: 1.2$. Vulva with elevated lips, situated just posterior to oesophagointestinal junction; strongly muscular vagina directed posteriorly from vulva, 193 (175-213) long, containing eggs arranged in single row. Eggs elongate, with slightly protruding polar plugs and uncleaved content, 60 (55-62) long and 23 (20-26) wide; polar plugs 5-6 long, 5-6 wide; egg wall thick (2.0-2.5), comprising 3 layers, inner hyaline layer, darker middle layer, and outer layer forming fine coating with irregular sculpture; latter only visible at both egg ends. Ovary extends posteriorly to approximately distal end of intestine, 65 (35-145) from caudal end. Rectum 46 (30-55) long. Caudal end rounded; anus subterminal. Tail 4 (3-5) long.

Type host: Nemadactylus bergi (Norman) (syn. Cheilodactylus bergi) (Latridae, Perciformes)

Site: Stomach.

Type locality: SW Atlantic off Mar del Plata, $38^{\circ} 08^{\prime} \mathrm{S}$, $57^{\circ} 32^{\prime} \mathrm{W}$, Buenos Aires Province, Argentina.

Date of collection: May-June, 2008.

Deposition of types: Holotype: 1 male (C.H.M.L.P. Coll. No. 5878); allotype: 1 female (C.H.M.L.P. Coll. No. 5879); paratypes: 5 males and 5 females (C.H.M.L.P. Coll. No. 5880); also 5 males and 5 females in the Helminthological Collection of the Institute of Parasitology, Biology Centre, ASCR, České Budějovice (Coll. No. N-908).

Prevalence: $81.8 \%$.

Mean intensity \pm SD (range): $12.4 \pm 10.3(1-40)$.

Etymology: The specific name is from Greek (ákantha $=$ spine + kótylos $=$ cup) and refers to the spinose cupshaped basal portion of the spicular sheath.

\section{DISCUSSION}

The morphological characters of the newly found species agree with the diagnosis of Paracapillaroides (see Moravec et al. 1999), to which it is considered to belong, especially in relation to the fact that it has a spicular sheath consisting of a wider and shorter spinose proximal part and a longer aspinose distal part, which is unique among capillariids (Moravec et al. 1999).

The new species is readily distinguishable from $P$. agonostomi, the only previously known species of the genus, by having in both sexes a longer oesophagus relative to the body length (50-75\% of total body length vs. $22-48 \%$ in $P$. agonostomi). Males of the new species have a shorter and more complex spicule, forming approximately 3-5\% of the total body length (vs. $12-13 \%$ in P. agonostomi), and a more complex morphology of the spicular sheath. 

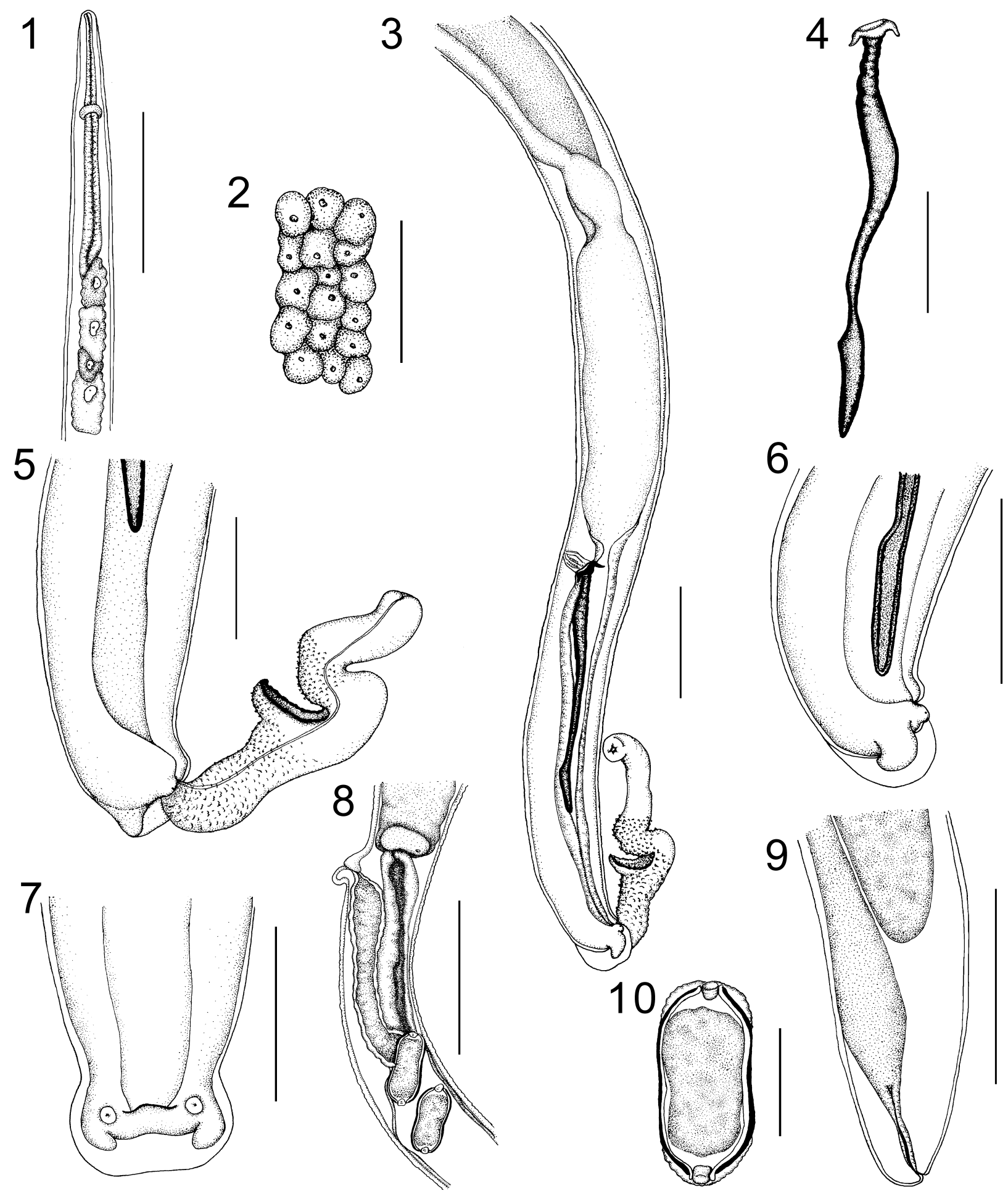

Figs. 1-10. Paracapillaroides acanthocotylus sp. n. Fig. 1. Anterior end of female, lateral view. Fig. 2. Bacillary band of female, at level of stichosome. Fig. 3. Posterior end of male, lateral view. Fig. 4. Spicule. Fig. 5. Spicular sheath, lateral view. Fig. 6. Tail of male, lateral view. Fig. 7. Tail of male, ventral view. Fig. 8. Female, region of vulva, lateral view. Fig. 9. Tail of female, lateral view. Fig. 10. Egg. Scale bars: Figs. $1,8=125 \mu \mathrm{m}$; Figs. 2, 5, 7, $10=30 \mu \mathrm{m}$; Figs. 3, 4, 6, $7=50 \mu \mathrm{m}$; Fig. $9=100 \mu \mathrm{m}$. 
The rays supporting the caudal bursa of males are also short and rounded, rather than digitiform, and females have elevated vulval lips and longer eggs.

The complex structure of both the spicule and the spicular sheath is unique among capillariids parasitizing coldblooded vertebrates. Based on these differences, a new species, Paracapillaroides acanthocotylus, is proposed. To our knowledge this is the first record of a capillariid nematode parasitizing a latrid fish.
Acknowledgements. The authors wish to thank Dr. František Moravec (Institute of Parasitology, Biology Centre of the Academy of Sciences of the Czech Republic, Ceské Budějovice) for his useful review of an early version of the manuscript and $\mathrm{Mr}$. Roberto Mazella and Mr. Hugo Mazella from the fish market Albatros, Mar del Plata, for kindly providing fish samples. The present study was funded by grants from CONICET (PIP No. 5996) and Universidad Nacional de Mar del Plata (EXA 442/08).

\section{REFERENCES}

Moravec F. 2001: Trichinelloid Nematodes Parasitic in Coldblooded Vertebrates. Academia, Prague, 429 pp.

Moravec F., Salgado-Maldonado G., Caspeta-Mandu-jano J. 1999: Capillariids (Nematoda: Capillariidae) from Agonostomus monticola and Gobiomorus spp. (Pisces, Mugilidae and Eleotridae) from fresh waters in Mexico. Acta Parasitol. 44: 180-187.
Timi J.T., Rossin M.A., Lanfranchi A.L. 2006: A new species of Capillaria (Nematoda: Capillariidae) parasitizing Conger orbignianus (Pisces: Congridae) from Argentina. J. Parasitol. 92: 628-630.

Timi J.T., Rossin M.A., Lanfranchi A.L., Etchegoin J.A. 2007: A new species of Paracapillaria (Nematoda: Capillariidae) parasitizing the Brazilian sandperch Pinguipes brasilianus (Pisces: Pinguipedidae) from Argentina. J. Parasitol. 93: 922-924.

Accepted 23 September 2008 